
\title{
3 Research Square \\ The Zone II Aorta is not a Forbidden Zone for Occlusion in Women with Morbidly Adherent Placenta
}

Juanfang Liu

The First Affiliated Hospital of Zhengzhou University

Shanshan Xie

The First Affiliated Hospital of Zhengzhou University https://orcid.org/0000-0002-7970-6705

Xueliang Zhou

The First Affiliated Hospital of Zhengzhou University

Zhaonan Li

The First Affiliated Hospital of Zhengzhou University

Jianjian Chen

The First Affiliated Hospital of Zhengzhou University

Xinwei Han ( $D 1021056466 @ q q . c o m$ )

Zhengzhou University First Affiliated Hospital https://orcid.org/0000-0002-8090-9876

\section{Research Article}

Keywords: balloon catheter, middle abdominal aorta, zone II aorta, morbidly adherent placenta, massive haemorrhage

Posted Date: August 26th, 2021

DOl: https://doi.org/10.21203/rs.3.rs-159184/v1

License: (c) (i) This work is licensed under a Creative Commons Attribution 4.0 International License. Read Full License 


\section{Abstract}

Aim: To evaluate the safety and efficacy of balloon occlusion at the Zone II aorta for the management of morbidly adherent placenta.

Methods: From September 2015 to October 2018, a total of 80 consecutive patients who were prenatally diagnosed with morbidly adherent placenta were assigned into two groups: the balloon occlusion group $(n=40)$ and the non-balloon occlusion group $(n=40)$.

The intraoperative estimated blood loss, blood transfusion, urine output, serum creatinine (Scr), blood urea nitrogen (BUN) and hysterectomy rate were recorded and compared between the two groups.

Results: The estimated blood loss in the balloon occlusion group was significantly lower than that in the non-balloon occlusion group $(811.75 \pm 299.93 \mathrm{ml}$ vs $1529.75 \pm 808.01 \mathrm{ml}, \mathrm{P}<0.001)$. The median amount of packed RBCs transfused in the balloon occlusion group and non-balloon occlusion group was $0 \mathrm{U}$ and 2 $\mathrm{U}$, respectively $(\mathrm{P}=0.001)$. The women in the former group had a lower blood transfusion rate than those in the latter group ( $30 \%$ vs $57.5 \%, P=0.013)$. Hysterectomy occurred in none in the balloon occlusion group but in 6 patients in the non-balloon occlusion group $(P=0.011)$.

Conclusion: The middle abdominal aorta (Zone II) is not a forbidden zone for occlusion as long as the single occlusion time is limited to 15 mins. Balloon occlusion at the Zone II aorta can effectively reduce blood loss, transfusion requirements and hysterectomy rates in patients with morbidly adherent placenta.

\section{Introduction}

Morbidly adherent placenta (MAP) remains a major cause of the most severe postpartum haemorrhage and maternal mortality in the world. MAP includes placenta accreta, increta, and percreta, which refer to the status of placental villous invasion of the myometrium and serosa or even attachment to adjacent organs such as the bladder. If placental tissue cannot be completely separated from the uterus during caesarean section, massive bleeding often leads to poor visualization of the operation field, coagulopathy, disseminated intravascular coagulopathy (DIC), uterine hysterectomy and even maternal death $^{[1,2]}$. Caesarean hysterectomy remains the mainstream management approach to reduce the incidence of maternal death ${ }^{[3]}$.

Recently, the use of endovascular technology in dealing with MAP has grown to be an important part of the surgical plans to control intraoperative bleeding and preserve the uterus. The value of temporary bilateral occlusion of the common iliac arteries and internal iliac arteries in controlling intraoperative bleeding has been demonstrated by many researchers ${ }^{[4,5]}$. Furthermore, some authors advocate temporary balloon occlusion of the abdominal aorta owing to its impressive results in reducing the estimated blood loss and hysterectomy rate ${ }^{[6,7]}$. Manzano-Nunez $\mathrm{R}$ et al. performed a systematic review and concluded that resuscitative endovascular balloon occlusion of the aorta (REBOA) was a feasible therapeutic strategy option for prophylactic haemorrhage control in 336 pregnant women with MAP 
undergoing elective caesarean delivery ${ }^{[8]}$. Many studies have verified the safety and efficacy of caesarean delivery with prophylactic zone III aortic REBOA occlusion in women with MAP. REBOA has reappeared as a safe and effective intervention not only in the management of torso haemorrhage but also in the control of massive haemorrhage in cases of $\mathrm{MAP}^{[8,9]}$.

The aortic occlusion area is often divided into three areas: zone I, from the left subclavian artery opening to the celiac artery (upper abdominal aorta); zone II, from the celiac artery to the lowest renal artery (middle abdominal aorta); and zone III, from the lowest renal artery to the bifurcation of the abdominal aorta (lower abdominal aorta). At present, there are many disputes regarding the clinical application of balloon occlusion of the abdominal aorta. Scholars generally believe that the middle abdominal aorta is not suitable for occlusion, as there are many branches of visceral blood vessels. A previous study we performed showed that less blood loss in the group of prophylactic balloon occlusion of the abdominal aorta (PBOA) at the level of the renal artery than the group of PBOA at the zone III ${ }^{[10]}$. The natural collateral circulations to the uterus such as ovarian artery, rectal, external iliac and inferior mesenteric arteries have been reported by many scholars ${ }^{[11-16]}$.

In clinical practice, there is no uniform standard for the duration of blood flow occlusion. A longer period of balloon occlusion will cause ischemia-reperfusion injury (IRI), which will lead to organ failure. However, a shorter period of occlusion will affect the procedure of operation and increase the amount of blood loss. Funahashi $Y$ demonstrated that irreversible damage occurred when ischemia time $\geq 25 \mathrm{~min}^{[17]}$. In the present study, we wanted to verify the safety and efficacy of zone II aortic balloon occlusion for $15 \mathrm{~min}$ in patients with morbidly adherent placenta. Data on renal function, estimated blood loss, packed $\mathrm{RBC}$ transfusion and uterus conservation rate were collected and analysed.

\section{Materials And Methods}

From September 2015 to October 2018, a total of 80 patients diagnosed with MAP in our tertiary referral centre were assigned into two groups: the balloon occlusion group $(n=40)$ and the non-balloon occlusion group $(n=40)$. Women in the balloon occlusion group had temporary prophylactic zone II aortic balloon occlusion performed prior to caesarean section. The inclusion criteria were as follows: (a) prenatal diagnosis of morbidly adherent placenta by Doppler ultrasonography or MRI, confirmed by intraoperative or postoperative pathologic examination; (b) pregnancy length more than 32 weeks; (c) complete clinical data; and (d) desire to preserve fertility. Informed consent was obtained from all patients and their families before surgery. The study conformed to Declaration of Helsinki and was approved by the institutional ethics committee. Written informed consent was obtained from all of the patients before therapy. Given the retrospective nature of the study and the use of anonymized data, requirements for informed consent were waived.

\section{Procedure}


All procedures were carried out in a hybrid operation room equipped with a C-arm for digital subtraction angiography (DSA). The same multidisciplinary team performed all the obstetric procedures and radiological investigations. General or epidural anaesthesia without premedication was administered as deemed appropriate by the anaesthesiologist ${ }^{[12]}$. First, an 8-F arterial sheath (Bard Peripheral Vascular Inc., USA) was placed in the right common femoral artery with the use of the Seldinger technique. Then, angiography was performed through a 5-F pigtail catheter (Cook, Bloomington, IN, USA) to locate the origin of the renal arteries. The commonly used balloon catheters with sizes of $8 \mathrm{~F}, 40 \times 16 \mathrm{~mm}$ and $40 \times$ $18 \mathrm{~mm}$ (Bard Peripheral Vascular, Tempe, AZ, USA) were introduced into the Zone II aorta under fluoroscopic guidance, and the upper tip of the balloon was placed at the origin of the upper renal artery. As soon as the baby was delivered, the occlusion balloons were inflated. Once the pulse oximeter decreased to 0 in the distal foot toe monitor, effective occlusion was obtained. With the aid of balloon occlusion, obstetricians were then able to separate the placenta from the uterine wall with bloodless visualization of the operating field. The single occlusion time was 15 minutes with a 1-minute interval. During this interval, the uterine cervix was blocked with an elastic tourniquet. If satisfactory haemostasis was not achieved, angiography of the uterine arteries was required, and uterine artery embolization was performed when necessary. Occasionally, ovarian artery embolization was performed to achieve haemostasis. Finally, the sheath was removed, and manual compression was applied to the puncture sites for at least 15 mins.

The procedures for caesarean section in both groups were the same. Some haemostasis methods, such as contractile drug injection, local suture ligation, uterine artery ligation and B-Lynch suture, were performed to achieve haemostasis during caesarean section. Given the high risk of delayed postpartum haemorrhage and pelvic infection, it is not recommended to leave the placenta in situ. The obstetrician then surgically excised as much of the placenta as possible along with the myometrium or serosa and restored the integrity of the uterus. If bleeding was not controlled after all of the methods above, a subtotal hysterectomy was then performed.

\section{Outcome Measures}

The primary outcome was intraoperative blood loss. The secondary outcomes included the amount of packed RBCs transfused, serum creatinine, blood urea nitrogen, urinary volume, the rate of hysterectomy, foetal radiation exposure and maternal complications.

\section{Statistical analysis}

Statistical analysis was performed using SPSS 19.0 (SPSS Inc., Chicago, IL, USA). Continuous data are expressed by the mean \pm the SD or the median (range). Categorical variables are expressed as percentages. An independent-sample t-test or the Mann-Whitney $U$ test was used to evaluate intergroup differences. Categorical variables were expressed as percentages, and the chi-squared test or Fisher's exact test were used for determining the differences. $P<0.05$ was considered statistically significant. 


\section{Results}

The demographic and obstetric characteristics of the two groups were similar, as listed in Table 1. The mean ages were $32.75 \pm 3.78$ years and $32.47 \pm 4.19$ years in the balloon occlusion and non-balloon occlusion groups, respectively, $P>0.05$. The gestational ages did not differ $(35.63 \pm 1.11$ wks vs $35.51 \pm$ 1.18 wks, $P>0.05$ ). The types of MAP were 15,15 , and 10 cases of placenta accrete, increta and percreta, respectively, in the balloon occlusion group and 16,17 and 7 cases, respectively, in the non-balloon occlusion group $(P>0.05)$.

Table 1

Demographic and obstetric characteristics of the two groups

\begin{tabular}{|llll|}
\hline & Balloon group $(n=40)$ & Non-balloon group $(n=40)$ & $P$ \\
\hline Age $(\mathrm{y})$ & $32.75 \pm 3.78$ & $32.47 \pm 4.19$ & 0.780 \\
\hline Gestational age $(\mathrm{wk})$ & $35.63 \pm 1.11$ & $35.51 \pm 1.18$ & 0.649 \\
\hline Weight gain during pregnancy $(\mathrm{kg})$ & $14.32 \pm 3.87$ & $13.97 \pm 3.97$ & 0.691 \\
\hline BMI $\left(\mathrm{kg} / \mathrm{m}^{2}\right)$ & $26.65 \pm 2.03$ & $27.36 \pm 1.62$ & 0.091 \\
\hline Gravidity & $2.5(2-3)$ & $2.5(2-3)$ & 0.836 \\
\hline Prior caesarean section & $1(0-1)$ & $1(0-1)$ & 0.884 \\
\hline Parity & $0.5(0-1)$ & $0.5(0-1)$ & $>0.999$ \\
\hline Placental types & & 16 & 0.709 \\
\hline accreta & 15 & 17 & \\
increta & 15 & 7 & \\
\hline percreta & 10 & 16 & \\
\hline
\end{tabular}

The estimated blood loss in the balloon occlusion group was significantly lower than that in the nonballoon occlusion group $(811.75 \pm 299.93 \mathrm{~mL}$ vs $1529.75 \pm 808.01 \mathrm{~mL}, \mathrm{P}<0.001)$. The median amount of packed RBCs transfused in the balloon occlusion group and non-balloon occlusion group was $0 \mathrm{U}$ and 2 $U$, respectively $(P=0.001)$. The women in the balloon occlusion group had a lower blood transfusion rate than those in the non-balloon occlusion group ( $30 \%$ vs $57.5 \%, P=0.013)$. Subtotal hysterectomy was performed in 6 patients $(15 \%)$ in the non-balloon occlusion group but in none $(0 \%)$ in the balloon occlusion group, indicating a significant difference $(P=0.011)$. However, no significant intergroup differences were noted in terms of serum creatinine (Scr) and blood urea nitrogen (BUN) $(P=0.365$ and 0.634 , respectively). Urine output was $997.00 \pm 344.75 \mathrm{ml}$ and $968.75 \pm 358.15 \mathrm{ml}$ in the balloon and nonballoon occlusion groups, respectively, and no intergroup differences existed $(P>0.05)$. There was no difference in mean birth weight or Apgar score $(>7)$ at 1 min between the two groups (all $P>0.05$ ). In the balloon occlusion group, the foetal radiation exposure was $5.05 \pm 1.08 \mathrm{mGy}$, and one woman had 
thrombosis in the right femoral artery and recovered after thrombolysis and anticoagulation therapy before discharge. No other complications related to balloon occlusion occurred in this study (Table 2).

Table 2

Patient characteristics and outcomes

\begin{tabular}{|llll|}
\hline characteristic & Balloon group $(\mathrm{n}=\mathbf{4 0})$ & Non-balloon group $(\mathrm{n}=40)$ & $\mathbf{P}$ \\
\hline Estimated blood loss $(\mathrm{mL})$ & $811.75 \pm 299.93$ & $1529.75 \pm 808.01$ & $<0.001$ \\
\hline Blood transfusion & $12(30 \%)$ & $23(57.5 \%)$ & 0.013 \\
\hline Packed RBCs transfused $(\mathrm{U})$ & $0(0-2)$ & $2(0-4)$ & 0.001 \\
\hline Hysterectomy & 0 & $6(15 \%)$ & 0.011 \\
\hline Serum creatinine level $(\mu \mathrm{mol} / \mathrm{L})$ & $36.00 \pm 3.86$ & $35.15 \pm 4.45$ & 0.365 \\
\hline Blood urea nitrogen $(\mathrm{mmol} / \mathrm{L})$ & $3.35 \pm 0.82$ & $3.43 \pm 0.64$ & 0.634 \\
\hline Urine output $(\mathrm{mL})$ & $997.00 \pm 344.75$ & $968.75 \pm 358.15$ & 0.720 \\
\hline Apgar score $>7$ at 1 min & 38 & $40(100 \%)$ & 0.093 \\
\hline Mean birth weight $(\mathrm{g})$ & $2611.50 \pm 365.20$ & $2678.00 \pm 328.26$ & 0.394 \\
\hline Foetal radiation exposure & $5.05 \pm 1.08 \mathrm{mGy}$ & - & \\
\hline Thrombosis in RFA & 1 & - & \\
\hline
\end{tabular}

All 80 women received telephone follow-up for 12-15 months after delivery. In all patients, the uterus was gradually restored to the same size as before, as determined through ultrasonography. In all patients, no postpartum infection or vaginal bleeding occurred, and menstrual cycles were all restored.

\section{Discussion}

Placenta accreta represents a main cause of obstetric massive haemorrhage. Although conservative management, such as intrauterine tamponade and B-Lynch suture, is effective to some extent, caesarean hysterectomy remains the mainstream treatment to reduce maternal mortality ${ }^{[3]}$. Unfortunately, this method results in a permanent loss of fertility for these women.

Abdominal aortic balloon occlusion has gained superiority in reducing blood loss and blood transfusion compared with internal iliac and common iliac artery balloon occlusion ${ }^{[14]}$. Our previous study revealed that the rate of postpartum bleeding originating from the ovarian arteries reached $19.35 \%{ }^{[18]}$. In addition, the placement of balloon catheters at the zone II aorta has an advantage over placement at the zone III aorta in reducing intraoperative blood loss and the rate of ovarian artery embolization ${ }^{[19]}$. Given the bilateral anatomical distribution of the ovarian arteries, balloon catheters were placed at the midpoint between the left and right renal arteries in the observation group. In a majority of cases, the left ovarian artery originates from the left renal artery or the anterior lateral wall of the abdominal aorta, whereas the 
right ovarian artery originates from the abdominal aorta ${ }^{[20]}$. Therefore, blood flow from the ovarian arteries, including other ectopic arteries, can be temporarily blocked when the aortic balloon is placed at the zone II aorta. Our previous study showed that retaining the sheath was of little use because the risk of rebleeding was very low. Meanwhile, the risk of arterial thrombosis on the target leg was reduced, so we removed the sheaths immediately after surgery. Patients in the balloon occlusion group were monitored for the right leg temperature, blood pressure and pulsation of the dorsal artery of the foot. Other monitoring measures were routinely except for vascular ultrasound examination of lower extremity.

In our study, balloon occlusion at the Zone II aorta played an important role in reducing the amount of bleeding during caesarean section, $811.75 \mathrm{~mL}$ in the balloon occlusion group and $1529.75 \mathrm{~mL}$ in the control group. The amount of blood loss in the present study was less than which $(1560.5 \mathrm{~mL})$ reported by Cui $\mathrm{S}$, et al conducted balloon occlusion at the Zone II aorta ${ }^{[11]}$. The uterus was conserved in all 40 patients $(100 \%)$ in the observation group, but in the control group, 6 women $(15 \%)$ received subtotal hysterectomy.

Postoperative evaluation showed that there was no significant difference in BUN, Scr or urine output between the two groups. None of the patients developed renal dysfunction. In this study, the single block time was restricted to 15 minutes to avoid complications owing to long-term occlusion caused by the balloon catheter. Yasuhito et al. found that a single block time of no more than 25 minutes will not cause damage to the kidneys ${ }^{[17]}$. In the present study, no cases of severe complications were observed. Only one case of thrombosis in the right femoral artery was observed in the conservation group, with up to 3 punctures in this patient due to obesity. We assume that fewer punctures and a shorter operation time may help prevent complications to some degree. We regard the foetal exposure of $5.05 \mathrm{mGy}$ in this study as relatively safe; as long as the radiation exposure is no more than $150 \mathrm{mGy}$, there are no adverse effects on the foetus ${ }^{[21]}$.

\section{Limitations}

It should be noted that there are limitations in our study. Firstly, this was a retrospective study with a limited number of patients in single centre. Thus, prospective, randomized and multicentre controlled trials with a larger number of participants are needed to verify the efficacy and safety of zone II aortic balloon occlusion.

\section{Conclusions}

In brief, the present study revealed that prophylactic balloon occlusion at the zone II aorta is relatively safe and represents a reasonable treatment for reducing haemorrhage in placenta accrete patients.

\section{Declarations}

\section{Data Availability}


The data used to support the findings of this study are available from the corresponding author upon request.

Funding There is no funding related to this study.

Conflict of interest The authors have no conflicts of interest to declare.

\section{References}

Primary Sources

Secondary Sources

Uncategorized References

1. Yu, S. C. H., Cheng, Y. K. Y., Tse, W. T., Sahota, D. S., Chung, M. Y., Wong, S. S. M., Chan, O. K., and Leung, T. Y. (2020) Perioperative prophylactic internal iliac artery balloon occlusion in the prevention of postpartum hemorrhage in placenta previa: a randomized controlled trial, American journal of obstetrics and gynecology.

2. Practice, C. o. O. (2012) Placenta accreta. Committee Opinion No. 529. American College of Obstetricians and Gynecologists., OBSTETRICS \& GYNECOLOGY 120, 207-211.

3. Bailit, J. L., Grobman, W. A., Rice, M. M., Reddy, U. M., Wapner, R. J., Varner, M. W., Leveno, K. J., lams, J. D., Tita, A. T., Saade, G., Rouse, D. J., Blackwell, S. C., Eunice Kennedy Shriver National Institute of Child, H., and Human Development Maternal-Fetal Medicine Units, N. (2015) Morbidly adherent placenta treatments and outcomes, Obstet Gynecol 125, 683-689.

4. Feng, S., Liao, Z., and Huang, H. (2017) Effect of prophylactic placement of internal iliac artery balloon catheters on outcomes of women with placenta accreta: an impact study, Anaesthesia 72, 853858.

5. Allison, S. K., Ingraham, C., Aarabi, S., and Kogut, M. (2017) latrogenic Common Iliac Artery Rupture from Resuscitative Endovascular Balloon Occlusion of the Aorta, J Vasc Interv Radiol 28, 619-620.

6. Panici, P. B., Anceschi, M., Borgia, M. L., Bresadola, L., Masselli, G., Parasassi, T., Perrone, G., Fetal Maternal Risk, G., and Brunelli, R. (2012) Intraoperative aorta balloon occlusion: fertility preservation in patients with placenta previa accreta/increta, The journal of maternal-fetal \& neonatal medicine : the official journal of the European Association of Perinatal Medicine, the Federation of Asia and Oceania Perinatal Societies, the International Society of Perinatal Obstet 25, 2512-2516.

7. Qiu, Z., Hu, J., Wu, J., and Chen, L. (2017) Prophylactic temporary abdominal aorta balloon occlusion in women with placenta previa accretism during late gestation, Medicine 96, e8681. 
8. Manzano-Nunez, R., Escobar-Vidarte, M. F., Naranjo, M. P., Rodriguez, F., Ferrada, P., Casallas, J. D., and Ordonez, C. A. (2017) Expanding the field of acute care surgery: a systematic review of the use of resuscitative endovascular balloon occlusion of the aorta (REBOA) in cases of morbidly adherent placenta, European journal of trauma and emergency surgery : official publication of the European Trauma Society.

9. Sato, R., Kuriyama, A., Takaesu, R., Miyamae, N., Iwanaga, W., Tokuda, H., and Umemura, T. (2018) Resuscitative endovascular balloon occlusion of the aorta performed by emergency physicians for traumatic hemorrhagic shock: a case series from Japanese emergency rooms, Critical care 22, 103.

10. Liu, J., Xu, J., Jiao, D., Duan, X., and Han, X. (2019) Comparison of the efficacy of prophylactic balloon occlusion of the abdominal aorta at or below the level of the renal artery in women with placenta accreta undergoing cesarean section, The journal of maternal-fetal \& neonatal medicine : the official journal of the European Association of Perinatal Medicine, the Federation of Asia and Oceania Perinatal Societies, the International Society of Perinatal Obstet, 1-8.

11. Cui, S., Zhi, Y., Cheng, G., Zhang, K., Zhang, L., and Shen, L. (2017) Retrospective analysis of placenta previa with abnormal placentation with and without prophylactic use of abdominal aorta balloon occlusion, International Journal of Gynecology \& Obstetrics 137, 265-270.

12. Wei, X., Zhang, J., Chu, Q., Du, Y., Xing, N., Xu, X., Zhou, Y., and Zhang, W. (2016) Prophylactic abdominal aorta balloon occlusion during caesarean section: a retrospective case series, International journal of obstetric anesthesia 27, 3-8.

13. Chen, M., and Xie, L. (2016) Clinical evaluation of balloon occlusion of the lower abdominal aorta in patients with placenta previa and previous cesarean section: A retrospective study on 43 cases, International journal of surgery 34, 6-9.

14. Wang, Y. L., Duan, X. H., Han, X. W., Wang, L., Zhao, X. L., Chen, Z. M., Chu, Q. J., and Zhang, W. (2017) Comparison of temporary abdominal aortic occlusion with internal iliac artery occlusion for patients with placenta accreta - a non-randomised prospective study, VASA. Zeitschrift fur Gefasskrankheiten 46, 53-57.

15. Kim, J. E., So, Y. H., Kim, B. J., Kim, S. M., Choi, Y. H., and Sung, C. K. (2017) Postpartum hemorrhage from non-uterine arteries: clinical importance of their detection and the results of selective embolization, Acta radiologica 0, 1-7.

16. Wang, M. Q., Liu, F. Y., Duan, F., Wang, Z. J., Song, P., and Song, L. (2009) Ovarian artery embolization supplementing hypogastric-uterine artery embolization for control of severe postpartum hemorrhage: report of eight cases, J Vasc Interv Radiol 20, 971-976.

17. Funahashi, Y., Hattori, R., Yamamoto, T., Kamihira, O., Kato, K., and Gotoh, M. (2009) Ischemic renal damage after nephron-sparing surgery in patients with normal contralateral kidney, European urology 55, 
18. J, L., Y, W., D, J., W, Z., and X, H. (2019) Prophylactic Occlusion Balloon Placement in the Abdominal Aorta Combined with Uterine or Ovarian Artery Embolization for the Prevention of Cesarean Hysterectomy Due to Placenta Accreta: A Retrospective Study, Cardiovascular and interventional radiology 42, 829-834.

19. J, L., J, X., D, J., X, D., and X, H. (2019) Comparison of the efficacy of prophylactic balloon occlusion of the abdominal aorta at or below the level of the renal artery in women with placenta accreta undergoing cesarean section, The journal of maternal-fetal \& neonatal medicine, 1-8.

20. Bakheit, M. (2012) Bilateral origin of ovarian arteries from accessory renal arteries, Saudi Med J 33, 1122-1124.

21. Thabet, A., Kalva, S., Liu, B., Mueller, P., and Lee, S. (2012) Interventional radiology in pregnancy complications: indications, technique, and methods for minimizing radiation exposure, Radiographics 32, 255-274. 